
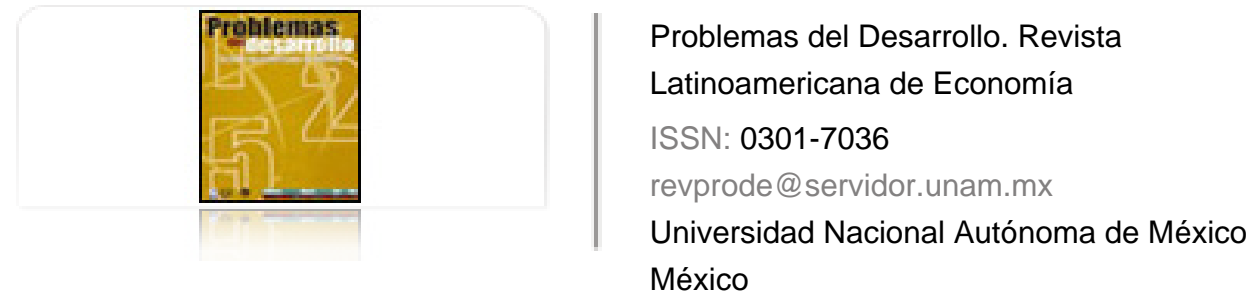

Gutiérrez Flores, Luis

La distribución del ingreso en México: un análisis regional, 1990-2004

Problemas del Desarrollo. Revista Latinoamericana de Economía, vol. 39, núm. 152, enero-marzo, 2008, pp. 139-163

Universidad Nacional Autónoma de México

Distrito Federal, México

Disponible en: http://www.redalyc.org/articulo.oa?id=11820668008

Cómo citar el artículo

Número completo

- Más información del artículo

Página de la revista en redalyc.org

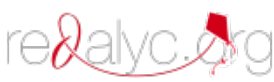

Sistema de Información Científica

Red de Revistas Científicas de América Latina, el Caribe, España y Portugal Proyecto académico sin fines de lucro, desarrollado bajo la iniciativa de acceso abierto 


\title{
LA DISTRIBUCIÓN DEL INGRESO EN MÉXICO: UN ANÁLISIS REGIONAL, 1990-2004
}

\author{
Luis Gutiérrez Flores* \\ Fecha de recepción: 7 de septiembre de 2007. Fecha de aceptación: 9 de diciembre de 2007.
}

\section{Resumen}

En este artículo se analiza la desigualdad regional del ingreso en México para el periodo 1990-2004 mediante la utilización de funciones de distribución del ingreso regional, junto con algunos indicadores de desigualdad. Para el análisis distribucional se han configurado distintas regiones con base en el diferente grado de exposición que han tenido las entidades federativas del país ante el proceso de apertura de la economía mexicana. En la construcción de las funciones regionales de distribución del ingreso tipo Gauss-kernel se han empleado datos del Producto Interno Bruto per capita por entidad federativa, junto con las proporciones de ingreso por estrato poblacional. La desigualdad es un fenómeno persistente en las regiones de México. Sin embargo, aquellas regiones que han estado más expuestas a los procesos de apertura económica presentan menores niveles de desigualdad. Lo contrario sucede con el resto de las regiones en ese mismo arreglo.

Palabras clave: distribución del ingreso, función de densidad, desigualdad regional, crecimiento económico.

\footnotetext{
Investigador-profesor del Centro de Investigaciones Socioeconómicas de la Universidad Autónoma de Coahuila. Correo electrónico: lugutier@ cise.uadec.mx.

Deseo expresar mi gratitud a Olga Sierra, David Castro y Gustavo Félix por el intercambio de ideas y las discusiones que motivaron este trabajo. De igual forma, agradezco los comentarios y sugerencias de dos dictaminadores anónimos. Sin embargo, cualquier error es responsabilidad exclusiva del autor.
} 
LUIS GUTIÉRREZ FLORES

140

Summary

This article analyzes regional income inequality in Mexico for the period 1990-2004 by utilizing regional income distribution functions, together with some inequality indicators. For the distribution analysis, distinct regions have been established based on different degrees of exposure experienced by the country's federal entities in the face of the Mexican economy's process of opening. In constructing regional income distribution functions of a Gauss-Kernel type, per capita Gross Domestic Product data by federal entity have been used, together with income proportions by population stratum. Inequality is a persistent phenomenon in Mexico's regions. However, some regions that have been more exposed to processes of economic opening show lower levels of inequality. The opposite occurs for the other regions in that same arrangement.

Key words: income distribution, density function, regional inequality, economic growth.

\section{Résumé}

Cet article analyse les différences de revenus au sein des régions du Mexique entre 1990 et 2004 en recourant à des fonctions de distribution du revenu régional auxquelles sont joints quelques indicateurs d'inégalité. Pour l'analyse distributionnelle, on a vu se configurer des régions distinctes selon le degré d'exposition des états de la République mexicaine au processus d'ouverture de son économie. Pour la construction des fonctions régionales de distribution du revenu type Gauss-Kernel, on a employé des données du Produit Interne Brut per capita par état fédéré, joints aux rapports de revenus par couche de population. L'inégalité est un phénomène persistant dans les régions du Mexique. Cependant, les régions qui ont été les plus exposées au processus d'ouverture économique présentent des niveaux plus faibles d'inégalité que les régions moins exposées.

Mots clés: distribution du revenu, fonction de densité, inégalité entre régions, croissance économique.

\section{Resumo}

Neste artigo analisa-se a desigualdade regional do ingresso no México no período 1990-2004 mediante a utilização de funções de distribuição do ingresso regional, junto com alguns indicadores de desigualdade. Para a análise distribucional configuraram distintas regiões com base no diferente grau de exposição que tenham tido as entidades federativas do país ante o processo de abertura da economia mexicana. Na construção das funções regionais de distribuição do ingresso tipo Gauss-kernel empregaram-se dados do Produto Interno Bruto per capita por entidade federativa, junto com as proporções de ingresso por estrato populacional. A desigualdade é um fenômeno persistente nas regiões do México. No entanto, aquelas regiões que estiveram mais expostas aos processos de abertura econômica apresentam menores níveis de desigualdade. O contrario acontece com o resto das regiões nesse mesmo esquema. Palavras-chave: distribuição do ingresso, função de densidade, desigualdade regional, crescimento econômico. 


\section{Introducción}

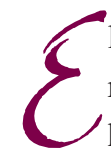

1 problema de la desigualdad económica se presenta como una de las características más importantes de la economía mexicana en los años recientes. De hecho, es un problema severo que de forma general ha recibido una creciente atención por parte de académicos y tomadores de decisiones. En este contexto, la medición de la desigualdad, las propiedades de la medición y sus efectos sobre el bienestar, y la relación que ésta mantiene con el crecimiento económico han sido algunos de los aspectos bajo la lupa. Usualmente, estos trabajos cuentan con un análisis generalizado en términos espaciales de la desigualdad económica y carecen de una revisión particularizada de la distribución del ingreso. Hay todavía una cantidad limitada de trabajos que describen qué hay detrás de la distribución del ingreso y, más aún, que exponen si es que la distribución cambia y en qué dirección lo hace. El análisis de la distribución del ingreso es importante, pues permite visualizar la magnitud de los cambios en la misma a lo largo del tiempo, lo cual inducirá cambios en los índices de desigualdad o de pobreza resultantes. Ello implica que en un contexto de apertura comercial y de liberalización económica es necesario conocer qué ha pasado con la distribución del ingreso de las diferentes regiones del país, así como la descripción del patrón de desigualdad de las mismas. La adhesión de México al Tratado de Libre Comercio de América del Norte (TLCAN) y el incremento en los flujos de Inversión Extranjera Directa (IED) han tenido impactos diferenciados sobre las regiones del país y, por tanto, sobre sus correspondientes distribuciones del ingreso que son importantes de evaluar en el contexto de las políticas públicas contemporáneas, así como también lo son para entender las consecuencias de la reestructuración económica en México. Aun las respuestas tentativas a estas cuestiones tienen importantes implicaciones de política territorial.

El resto del documento está organizado de la siguiente manera: en primera instancia se discute acerca de la pertinencia de las funciones de distribución del ingreso en los estudios de la desigualdad. Luego se expone la construcción de las funciones de distribución regional del ingreso junto con los resultados de los índices de desigualdad. En seguida ofrece algunos comentarios finales a la luz de los resultados obtenidos.

\section{Introducción al análisis de la desigualdad regional en México}

Si utilizamos como una medida del tamaño de una economía local al Producto Interno Bruto (PIB) per capita, una forma de abordar el análisis de la desigualdad es la obser- 
vación de qué tanto difieren las economías en cuanto al nivel que alcanzan de aquella variable. En particular, es interesante observar qué tan grande es una economía con respecto a otra. En la gráfica 1 se observa el número de veces que la economía más rica del país, el Distrito Federal, es mayor que la más pobre, la del estado de Chiapas.

Gráfica 1

Proporción del PIB per capita del DF sobre el PIB per capita de Chiapas en 1980-2004

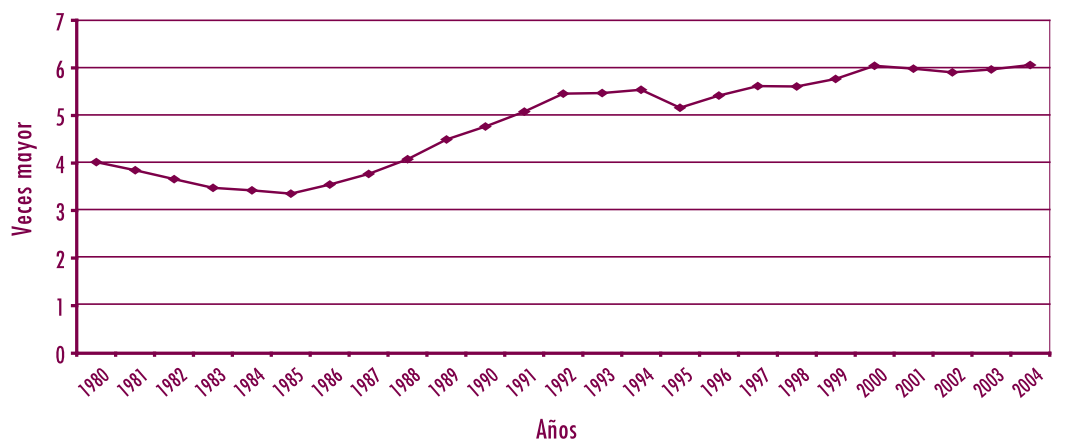

Fuente: Elaboración propia.

Aquí se desprenden dos importantes observaciones: en primer lugar, para los años de la década de los ochenta la diferencia entre la entidad más rica y la más pobre presentó una tendencia a la disminución y, en segundo lugar, dicha tendencia fue revertida desde finales de esa misma década y principios de los noventa. En este caso, la brecha entre estas economías representativas no solamente dejó de cerrarse, sino que se incrementó de forma sustancial. En los años recientes, la brecha alcanza un nivel de seis veces la diferencia en tamaño de la economía del Distrito Federal en relación con la economía de Chiapas.

Una preocupación persistente en la teoría del crecimiento económico ha sido la idea de qué hacer para cerrar la brecha entre las distintas economías. O en otras palabras, ¿qué fuerzas conducen a la convergencia entre las economías? Interesa averiguar si las diferencias interregionales de ingreso han tendido a incrementarse o a reducirse en el país. Sin duda, el análisis de la convergencia tiene rasgos ventajosos (Quah, 1996), como el hecho de que se trata de un análisis teóricamente atractivo, pues se puede mostrar que en muchos modelos la tasa de crecimiento del producto está relacionada con los niveles de ingreso mediante la contribución relativa del capital físico sobre el ingreso nacional. También este tipo de estudios permite estimar la velocidad a la que 
se acercan unas economías respecto de otras, al tiempo de dar a conocer la importancia de la acumulación del capital físico en la obtención del crecimiento económico. Sin embargo, los alcances de los estudios de convergencia son limitados cuando se trata de averiguar qué pasa hacia el interior de las economías en crecimiento.

Cuando las economías más pobres tienden a crecer a una mayor velocidad que las economías más ricas, decimos que hay convergencia- $\beta .{ }^{1}$ Adicionalmente, si la dispersión en el ingreso real per capita entre los grupos de la economía tiende a reducirse a lo largo del tiempo, decimos que hay convergencia- $\sigma$. Formalmente, la convergencia- $\sigma$ viene dada por la siguiente expresión (Sala-i-Martin, 1996):

$$
\sigma_{t}=(1 / n) \sum_{i=1}^{N}\left[\log \left(y_{i t}\right)-\mu_{t}\right]
$$

donde:

$i=1,2, \ldots, N$ es un grupo de regiones o economías,

$\mu_{t}=$ es la media muestral de $\log \left(y_{i t}\right)$,

$y_{i t}=$ es el ingreso real per capita de la región $i$ en el tiempo $t$, y

$\sigma_{t}=$ es la desviación estándar muestral de $\log \left(y_{i t}\right)$

Las siguientes dos gráficas muestran el análisis de convergencia- $\sigma$ para las entidades federativas de México. ${ }^{2}$ Dicho análisis se lleva a cabo en dos momentos: en primer lugar, para los años 1980-2004, que representan tanto el último tramo como el inicio de un cambio en la orientación de la economía mexicana que venía representada por un modelo de desarrollo hacia adentro del país; y, en segundo lugar, para los años 1994-2004, que muestra en particular a una economía con un modelo de desarrollo hacia el exterior a partir de la firma y entrada en vigor del Tratado de Libre Comercio de América del Norte.

En la gráfica 2 se observa que en los años ochenta se presentaba un proceso de convergencia entre las entidades del país, hecho que se denota por la reducción en la dispersión del PIB real per capita. La economía estaba homogeneizándose a un ritmo interesante. Sin embargo, casi al término de los ochenta este proceso empieza a revertirse. De hecho, la dispersión presenta un fuerte incremento en los primeros años de los noventa para mantenerse en esa dirección. Estos resultados coinciden con aquellos presentados en otros estudios, entre los que destacan el de Esquivel (1999), Messmacher (2000), Fuentes (2001), Esquivel y Messmacher (2002) y Chiquiar (2005).

1 Para más detalle, véase a Barro y Sala-i-Martin $(1992)$.
2 Una aplicación similar se encuentra en Ruiz $(2004,56)$. 
Gráfica 2

Convergencia Sigma del PIB per capita de 32 entidades federativas, México 1980-2004

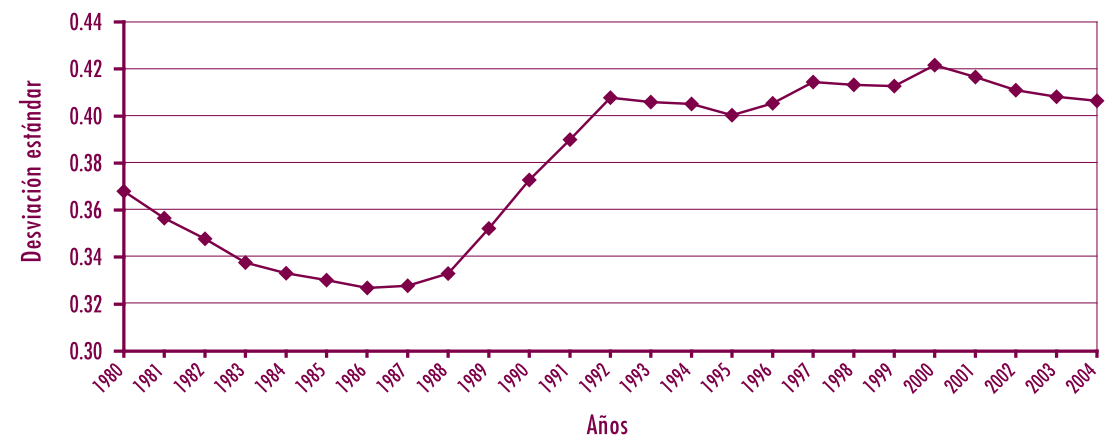

Fuente: Elaboración propia.

En general, estos trabajos tienen los siguientes rasgos comunes:

1. Todos tienden a concluir que hubo convergencia entre las entidades federativas de México hasta mediados de los ochenta, pero este proceso se detuvo en los años posteriores.

2. La unidad principal de análisis es el PIB por entidad federativa y sus correspondientes tasas de crecimiento.

Particularmente, Esquivel (1999) analiza las características del proceso de convergencia económica absoluta entre las regiones de México para el periodo 1940-1995. Para explicar la baja tasa de convergencia, discute acerca del papel que han desempeñado dos factores importantes en el desarrollo regional: la migración interestatal y el comportamiento regional de la educación. La evidencia obtenida por Messmacher (2000) conduce a dos situaciones: en primer lugar, hay un efecto sectorial, ya que el sector manufacturero se ha expandido en el caso de todas las entidades de la república, creciendo a una velocidad mayor que cualquier otro sector de actividad económica, con excepción del sector de transportes y comunicaciones. En segundo lugar, hay efecto regional, pues las entidades del norte han tenido un mejor desempeño económico. Sin embargo, el efecto sectorial parece dominar al efecto regional, ya que aquél es el principal determinante del desempeño relativo de los estados durante el periodo de análisis. Por su parte, Fuentes (2001) examina cómo algunos factores endógenos a las regiones, como el capital humano y la infraestructura pública, así como las economías externas (aglomeración y urbanización), han contribuido al proceso de convergencia (divergencia) regional observada en México a partir de la apertura comercial. Las causas que analiza para explicar dicha afirmación son las dotaciones de capital físico y humano, así como las economías externas. Más aún, 
Esquivel y Messmacher (2002) estudian las principales fuentes de convergencia regional en México entre 1960 y 2000. Sus resultados muestran que la productividad del trabajo fue un factor determinante para lograr la convergencia en el PIB per capita entre 1960 y 1990. Sin embargo, dicha tendencia no se materializó en los años sesenta y ochenta como un resultado en el comportamiento divergente en algunas variables del mercado de trabajo, como el empleo y la tasa de participación. Al contrario de esta tendencia, la productividad del trabajo se convirtió en la fuente principal del patrón de divergencia en el ingreso per capita que se observó durante la década de los noventa.

Chiquiar (2005) afirma que el patrón de divergencia observado después de 1985 no ha sido revertido con el TLCAN. Asimismo, los resultados sugieren también que los ganadores de las reformas han sido aquellos estados con una dotación inicial o asignada de mayores niveles de capital físico y humano, así como de una mejor infraestructura.

El punto de quiebre en el proceso de convergencia coincide con el proceso de ajuste heterodoxo seguido por las autoridades económicas del país, para la estabilización de precios y la aplicación de las medidas de cambio estructural, ${ }^{3}$ aun antes de la puesta en marcha del TLCAN. Las políticas de ajuste de precios vía la contención de la demanda agregada tuvieron como consecuencia la desaceleración de la economía en su conjunto, facilitando el proceso de divergencia económica. En todo caso, el proceso de convergencia ha sido ambiguo, y no se ha logrado que las economías estatales puedan converger a un ritmo deseado, o al menos sostenido.

Resulta interesante observar cómo se ha presentado el fenómeno de la convergencia en la década que siguió a la firma del TLCAN (gráfica 3).

Gráfica 3

Convergencia Sigma de las entidades federativas. México, 1990-2004

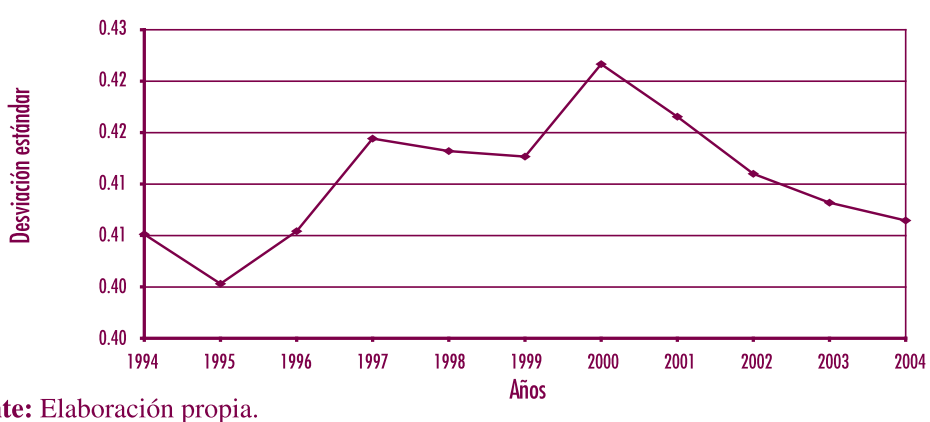

Fuente: Elaboración propia.

3 Véase Aspe (1993) capítulos 2, y 3 para una explicación más detallada del proceso de ajuste y de reforma. 
Es evidente que la crisis de 1995 repercutió de forma negativa sobre el proceso de convergencia como se observa en la gráfica. La recuperación económica que siguió a partir de ese año no contribuyó a reducir las diferencias entre las economías de las entidades federativas. Ello viene dado por un patrón de localización geográfica bien definido en favor de las entidades del norte del país. Más aún, a partir de 2000 y sobre todo en 2001, la desaceleración económica propició que dichas entidades ganadoras con el proceso de liberalización comercial redujeran su ritmo de crecimiento, lo que actuó en favor del proceso de convergencia.

Aún queda pendiente explorar la relación entre las economías de las entidades federativas desde el punto de vista de sus tasas de crecimiento. En general, se espera que las economías más pobres crezcan a tasas mayores que aquellas más ricas. Cuando esto sucede, se dice que hay un proceso de convergencia- $\beta$. Para distinguir entre los dos conceptos de convergencia, lo más conveniente es pensar en el grupo de economías bajo observación como una distribución dada del ingreso per capita. En todo caso, la convergencia- $\sigma$ dará cuenta de los movimientos totales de la distribución del ingreso mientras que la convergencia- $\beta$ indicará los movimientos internos en la distribución.

En el caso de las economías estatales de México, ${ }^{4}$ en primera instancia se presenta la relación que guarda el nivel de ingreso per capita en el año inicial con la tasa de crecimiento del PIB per capita:

Gráfica 4

Convergencia- $\beta$. PIB 1980 vs. tasa de crecimiento 1980-2004

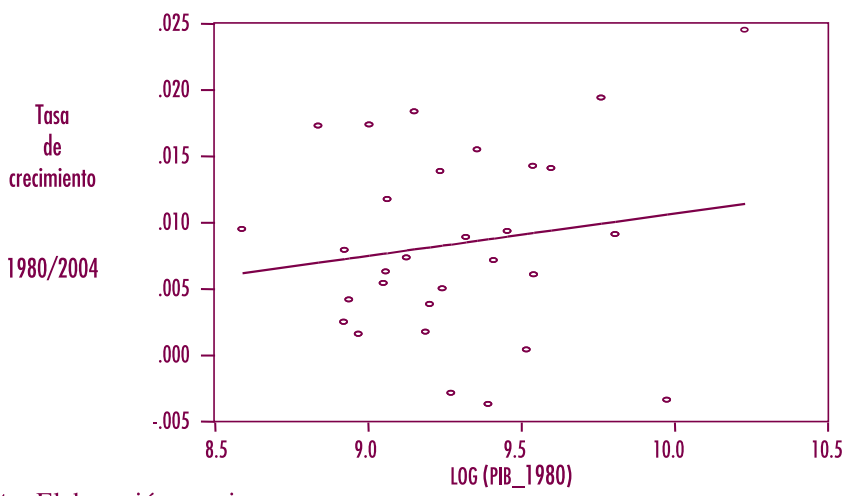

Fuente: Elaboración propia.

4 Cabe mencionar que en el análisis de la convergencia absoluta se excluyen los datos de Campeche y Tabasco, en virtud de que se trata de economías petroleras y se convierten en observaciones atípicas que pueden sesgar los resultados. Una explicación más detallada se encuentra en Esquivel (1999), pp. 737-738. 
Se espera que la relación entre el nivel inicial del PIB per capita y la tasa anual de crecimiento en el periodo sea negativa, indicando esto que efectivamente las economías pobres crecen más rápido que las economías más ricas, o que la distribución del PIB per capita se compacta cuando hay convergencia- $\beta$. Lo que se puede observar en la gráfica 4 es que ocurre exactamente lo contrario, la relación entre el PIB per capita inicial y la tasa de crecimiento de las entidades federativas en el periodo 1980-2004 presenta una tendencia positiva, lo cual permite afirmar no que hubo convergencia- $\beta$.

A continuación mostramos la convergencia- $\beta$ para el periodo más representativo de la liberalización comercial, el de 1994-2004.

Gráfica 5 Convergencia- $\beta$. PIB 1994 vs. tasa de crecimiento 1994-2004

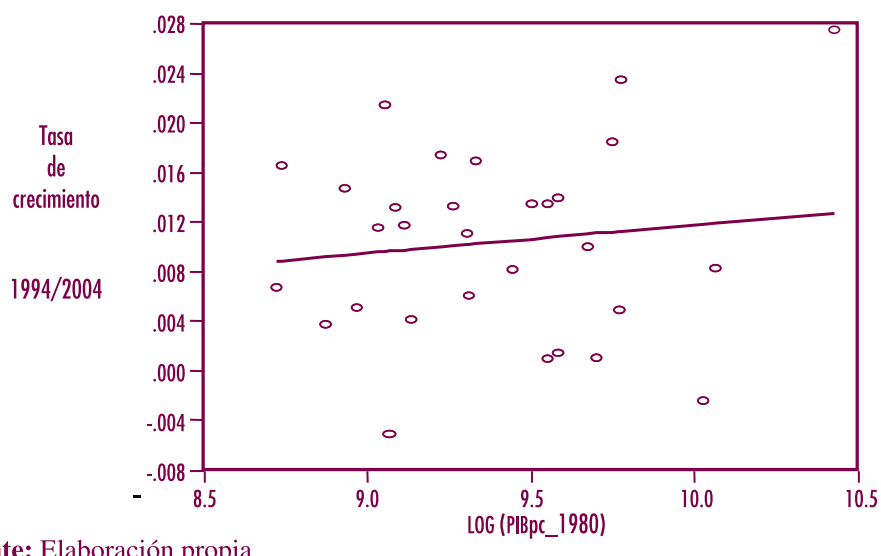

Fuente: Elaboración propia.

La relación positiva entre el nivel inicial de PIB per capita y la tasa de crecimiento de nuestro grupo de economías se mantiene para el periodo posterior a la liberalización del comercio. El proceso de divergencia es una característica del crecimiento económico en México. No se han presentado mecanismos o factores que contrarresten los efectos del cambio de modelo de desarrollo, de la crisis de 1995 y de la desaceleración económica experimentada en el año 2001.

También podemos estimar la velocidad de divergencia que se ha presentado en los periodos analizados para conocer la tasa a la cual se abre la brecha entre los estados del país. 
Para ello, utilizaremos la siguiente ecuación (Sala-i-Martin, 1996 y Chiquiar, 2005):

$$
\frac{1}{T} \log \left[\frac{y_{i, t_{0}+T}}{y_{i, t_{0}}}\right]=\alpha-\frac{1-e^{-\beta T}}{T} \log \left[y_{i, t_{0}}\right]+u_{i, t_{0} \cdot t_{o}+T}
$$

donde:

$y_{i, t_{0}}=$ Nivel inicial del producto per capita en el estado $i$ en el tiempo $t_{0}$,

$y_{i}, t_{0}+T=$ Nivel final del producto per capita en el estado $i$, después de que han transcurrido $T$ años.

$u_{i, t_{0} \cdot t_{o}+T}=$ Representa un promedio de los términos de error entre los tiempos $t_{0} \mathrm{y}$ $t_{0}+T$.

Los resultados se muestran en el siguiente cuadro:

Cuadro 1

Estimaciones de la tasa de convergencia absoluta para los estados.

(Variable dependiente: tasa de crecimiento promedio anual del PIBPc estatal)

\begin{tabular}{ccccc}
\multicolumn{5}{c}{ Tasa de Convergencia } \\
\hline Periodo & Coeficiente & Error estándar & $R^{2}$ & Observaciones \\
\hline $1980-2004$ & $-0.003072^{*}$ & 0.00123 & 0.25377 & 30 \\
$1994-2004$ & $-0.002246^{* *}$ & 0.00118 & 0.14818 & 30 \\
\hline
\end{tabular}

Fuente: Cálculos propios.

* Significativo a 5 por ciento

** Significativo a 10 por ciento

De la ecuación (2) observamos que bajo la hipótesis de convergencia absoluta, el parámetro $\beta$ debería presentar un signo positivo. La evidencia de un parámetro negativo sugeriría divergencia entre los niveles de producto per capita.

Los resultados soportan la hipótesis de divergencia absoluta para ambos periodos. ${ }^{5}$ Sin embargo, en el caso del periodo completo, 1980-2004, la velocidad de divergencia, aunque menor que en 1994-2004, permite inferir que las recurrentes crisis iniciadas en 1982 con el petróleo, pasando por 1987 en el sector financiero y llegando hasta 1995 con la crisis de la balanza de pagos, han profundizado las diferencias entre

5 En otros trabajos, como el de Esquivel (1999), se ha encontrado que la tasa de convergencia de la economía mexicana es de aproximadamente 1.2 por ciento para una serie de tiempo más amplia. 
las entidades federativas al tiempo que entre los años 1994 y 2004 el TLCAN no fue capaz de romper con dicha tendencia, que se ha visto reforzada.

\section{Estimación de funciones de distribución regional del ingreso}

En este apartado se presentan las funciones de distribución regional del ingreso desde su construcción hasta la estimación de sus correspondientes funciones de densidad para los años 1990, 1995, 2000 y 2004. Antes, se establece el criterio de regionalización utilizado en este estudio.

\section{Las regiones}

Las reformas comerciales de México parecen haber repercutido más en algunas partes del país que en otras (Hanson, 2005a). Hay regiones o entidades que han estado más expuestas a los efectos de la globalización. Para capturar estos efectos en términos de la distribución del ingreso y la desigualdad, utilizamos el siguiente criterio: la exposición a la globalización. Para ello, se emplea la razón que guardan dos variables consideradas como trascendentes en la reconfiguración económica que México ha experimentado a partir de la entrada en vigor del TLCAN, con respecto al PIB de las economías estatales. Estas variables son: la Inversión Extranjera Directa (IED) y el Valor Agregado de la Producción de la Industria Maquiladora de Exportación (VA).

Formalmente, el índice de exposición a la globalización se construye de la siguiente forma:

donde:

$$
I G_{i}=\frac{I E D_{i}}{P I B p c_{i}}+\frac{V A_{i}}{P I B p c_{i}}
$$

$I G_{i}=$ Índice de exposición a la globalización de la entidad $i$, $I E D_{i}=$ Inversión Extranjera Directa en la entidad $i$,

$V A_{i}=$ Valor Agregado de la Industria Maquiladora de Exportación de la entidad $i, \mathrm{y}$ $P I B P c_{i}=$ Producto Interno Bruto per capita de la entidad $i$.

El espíritu de este tipo de agrupamientos parece estar en línea con las ideas de autores como Hanson (2005a y 2005b) que clasifica a los grupos de entidades federativas en términos de la variación regional en la exposición a la inversión y a la composición de la estructura productiva, y Unger (2005) que utiliza franjas de entidades en relación con la distancia geográfica que éstas tienen de la frontera con Estados Unidos. La producción y el crecimiento del empleo de la industria maquiladora de exportación han ex- 
perimentado una marcada aceleración en su expansión con el advenimiento del TLCAN (Gruben, 2001). En México, el surgimiento de los flujos de IED se debe a varios factores económicos e institucionales (Ramírez, 2002) entre los que destacan: el programa de reconversión de la deuda, la privatización de sectores estratégicos y, desde luego, la liberalización del sector comercial. La IED genera las siguientes ventajas para las empresas transnacionales: en primer lugar, el establecimiento de las subsidiarias le otorga a las firmas madre la propiedad exclusiva sobre las patentes, las marcas y los procesos de producción. En segundo, se generan ventajas de localización de las subsidiarias por el acceso a mercados en crecimiento y con costos laborales más bajos, además de un reducido costo de transporte. Desde una perspectiva teórica, se ha tratado de establecer un nexo entre la IED y el crecimiento económico (Cuadros et al., 2004) cuando se analizan los efectos de la apertura sobre el desempeño económico.

Sin embargo, es obvio que la maquila y la IED no participan de igual forma en las economías regionales del país. Se considera entonces que ambas variables denotan en cierto grado la intensidad de cómo participan las distintas economías locales en la globalización y que, por tanto, comparten esta característica o no con otras entidades de la nación, y en función a ello se clasifican los distintos grupos de entidades. Es decir, se estima la ecuación (3) para cada entidad federativa y con base en el resultado se clasifica cada una de ellas. Así, las que han obtenido un valor más elevado del índice se identifican y agrupan en un sector denominado Región de Alta Exposición a la Globalización. Lo contrario sucede con el grupo de entidades que obtuvieron los valores más bajos del índice.

Así, en el siguiente cuadro se presentan las regiones que se utilizarán para los fines de este trabajo:

Cuadro 2

Regiones de México en función de su exposición a la globalización

\begin{tabular}{lcc}
\hline Alta exposición & Exposición media & Baja exposición \\
\hline Aguascalientes & Campeche & Colima \\
Baja California & Distrito Federal & Chiapas \\
Baja California Sur & Durango & Guerrero \\
Coahuila & Guanajuato & Hidalgo \\
Chihuahua & México & Michoacán \\
Jalisco & Morelos & Nayarit \\
Nuevo León & Querétaro & Oaxaca \\
Puebla & San Luis Potosí & Quintana Roo \\
Sonora & Tlaxcala & Sinaloa \\
Tamaulipas & Yucatán & Tabasco \\
& & Veracruz \\
& & Zacatecas \\
\hline
\end{tabular}

Fuente: Elaboración propia. 
Es necesario reconocer que tanto la IED como el VA no son los únicos factores que inciden sobre el desempeño de las regiones en el contexto de la globalización y los posibles cambios en la distribución del ingreso. Por ejemplo, el valor de las exportaciones y de las importaciones en relación con el producto per capita ayuda a estimar el grado de apertura de la economía, cosa que no sucede con la IED y el VA. Incluso hay actividades productivas en las entidades federativas que no están ligadas a la IED ni al VA, como los servicios a los productores que le imprimen una dinámica diferente a la economía local, pero que no se plasman en la magnitud de las variables elegidas. De hecho, esto sólo indica una parte de la historia. Sin embargo, la identificación de todos los factores que determinan el desempeño regional ante la exposición a los mercados globales aunque es importante, no es el objetivo de este trabajo.

\section{Metodología empírica}

El análisis empírico requiere la comparación de los cambios en la distribución regional del ingreso durante los años 1990, 1995, 2000 y 2004 entre los tres grupos de entidades o regiones. En este apartado describimos los enfoques utilizados para realizar dicha comparación.

Estimación de las proporciones de ingreso regional

Con información de los ingresos totales individuales ${ }^{6}$ de los últimos dos censos generales de población y vivienda (1990 y 2000) y del conteo de población y vivienda (1995) se han estimado las proporciones de ingreso por cada quintil de población, primero por entidad federativa y posteriormente por región.

Estas proporciones son necesarias para la estimación de la distribución regional del ingreso.

Las proporciones regionales de ingreso para 2004 se estimaron con el uso de un procedimiento econométrico. Dado que no se dispone de la información para realizar el cálculo de forma tradicional, se regresaron las proporciones obtenidas en los años anteriores en el tiempo y el valor de la ordenada se tomó como el valor de la proporción para cada quintil regional. ${ }^{7}$

6 En los censos, el ingreso total personal es obtenido de la suma de los siguientes rubros de ingreso: ingreso laboral, pensiones, remesas provenientes del exterior, remesas del interior, el ingreso del PROCAMPO o PROGRESA, y el ingreso del capital, en su definición más amplia. Véase a Cárdenas-Rodríguez, et al.,(2004) para una explicación más a fondo.

7 Para más detalle, véase Sala-i-Martin, 2002a, pp. 12-15. 
Estimación de las distribuciones

regionales del ingreso

Estamos ahora en condiciones de calcular las distribuciones regionales de ingreso por quintil de población. El procedimiento es el siguiente (Sala-i-Martin, 2002a): sea $N_{i t}$ la población total de la región $i$ en el tiempo $t$, a cada quinta parte de la población $\left(N_{i} / 5\right)$ se le asigna el ingreso $5 * S_{i k t} * Y_{i t}$, donde $S_{i k t}$ es la proporción de ingreso en la región $i$ del quintil $k$ en el tiempo $t$, y $Y_{i t}$ es el PIB per capita de la región $i$ en el tiempo $t$. Por lo tanto, se asume que dentro de cada quintil las personas tienen el mismo nivel de ingreso. ${ }^{8}$

La función kernel de densidad

Ahora podemos utilizar los datos de ingreso construidos en el apartado previo para estimar la distribución regional del ingreso. Estimamos una función de densidad (Silverman, 1986) tipo Gauss-kernel, que viene expresada de la siguiente forma:

$$
K=\frac{1}{\sqrt{2 \pi}} \exp \left(-\frac{1}{2} u^{2}\right)
$$

donde:

$K=$ función kernel

$u=$ argumento de la función kernel

Asimismo, se utiliza un ancho de banda dado por:

$$
h=0.9 * s d *\left(n^{-1 / 5}\right)=0.5695
$$

donde:

$h=$ ancho de banda de la función kernel ${ }^{9}$

$n=$ número de observaciones

$s d=$ desviación estándar del $\log (x)$

8 Es claro que esto implica que la desigualdad dentro de cada quintil no es tomada en cuenta. Sin embargo, Sala-i-Martin (2002a: 33-35) menciona que el sesgo por esta salvedad es en realidad poco significativo.

9 El ancho de banda de la función kernel, también conocido como "ancho de ventana", es el parámetro de suavizamiento que determina el ancho de los baches representados mediante el estimador kernel. Véase a Silverman (1986:15-17) para una explicación más detallada. 
En esta etapa del trabajo cabe aclarar que no se van a normalizar los datos. Esto con el fin de poder observar los movimientos laterales (hacia la derecha o izquierda) de la distribución y de esta forma inferir acerca de los cambios en la misma cuando el ingreso se incrementa o se reduce. A la vez, esto permite comparar varias distribuciones en el tiempo. ${ }^{10}$

A continuación, se presentan las estimaciones de las funciones de distribución regional del ingreso para cada año disponible:

Gráfica 6

Funciones de distribución regional del ingreso, 1990
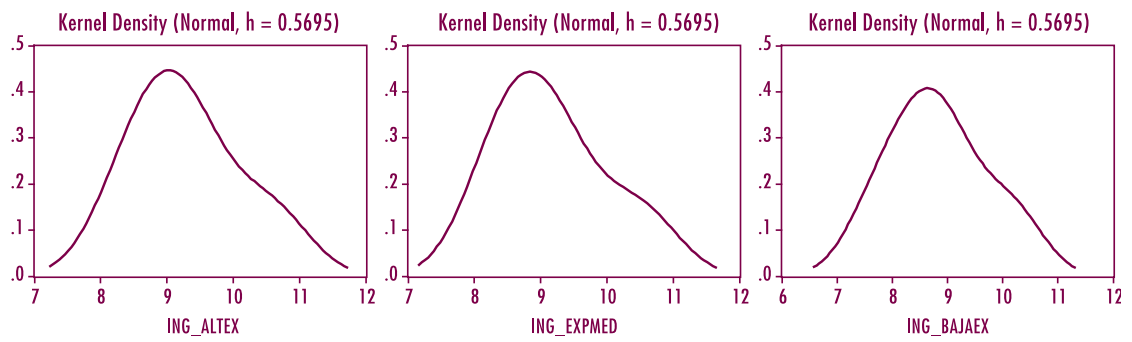

Fuente: Elaboración propia.

Lo primero que observamos de la gráfica 6 es que cada función de densidad regional tiene una diferente moda ${ }^{11} \mathrm{e}$ incluso hay casos en los que la distribución es bimodal. La moda de la función de densidad de la región de Alta Exposición a la Globalización (AEG) es de 8,104 pesos de 1993. La moda en la región de Exposición Media (EMG) es de 6,003 pesos y en la región de Baja Exposición (BEG) el valor de la moda alcanza un nivel de 4,024. En promedio, el nivel de ingreso de la región AEG es mayor al ingreso percibido por las regiones EMG y BEG. La función de densidad de la región AEG se sitúa más a la derecha que las funciones correspondientes a las regiones EMG y BEG. La densidad más baja se encuentra en el caso de la región BEG.

10 La normalización de datos no permite que la función de densidad se mueva hacia los lados, lo cual restringiría nuestro análisis. Adicionalmente, al no-normalizar estamos en posibilidades de estimar niveles de pobreza al integrar la función de densidad por detrás de un umbral. Para más detalle, véase a Quah (1997) y Sala-i-Martin (2002b).

11 La moda es el valor que más se repite dentro de la distribución dada. Cuando se da el caso de que dos valores diferentes aparecen como los más repetidos, se dice que la distribución es bimodal. Para más detalle, se puede consultar a Levin y Rubin (2004, pp. 85-86). 
Las regiones AEG y EMG presentan densidades muy similares. El sesgo que se aprecia por la forma de la función de distribución indica una mejor asignación del ingreso en el caso de las regiones AEG y EMG. En la región BEG hay una mayor tendencia a la concentración del ingreso.

Gráfica 7

Funciones de distribución regional del ingreso, 1995
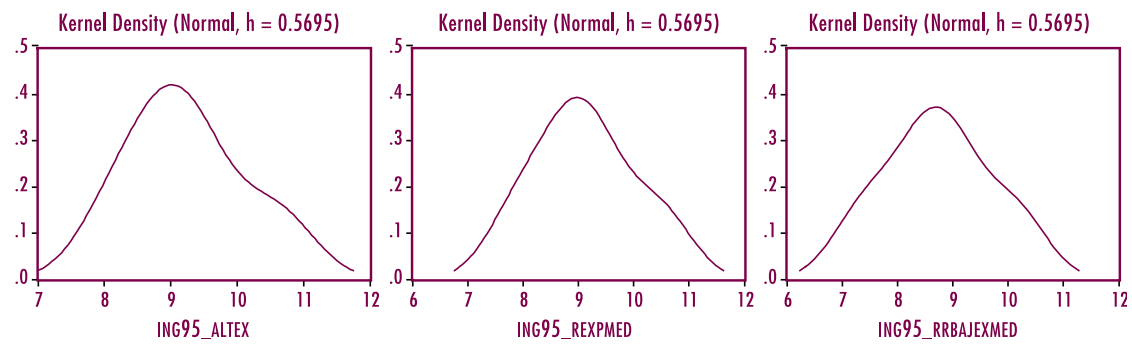

Fuente: Elaboración propia.

Para el año de 1995, la gráfica 7 muestra los resultados de haber construido las funciones regionales de distribución del ingreso. La moda de la región AEG se encuentra en el valor $\$ 8,955$, la de la región EMG es de $\$ 7,332$ y la de la región BEG es de $\$ 4,915$. Hubo un incremento en el ingreso promedio de las tres regiones analizadas entre los años 1990 y 1995. Sin embargo, la región EMG presenta un mayor incremento en su ingreso promedio (\$1,329 entre 1990 y 1995). La región con un

Gráfica 8

Funciones de distribución regional del ingreso, 2000
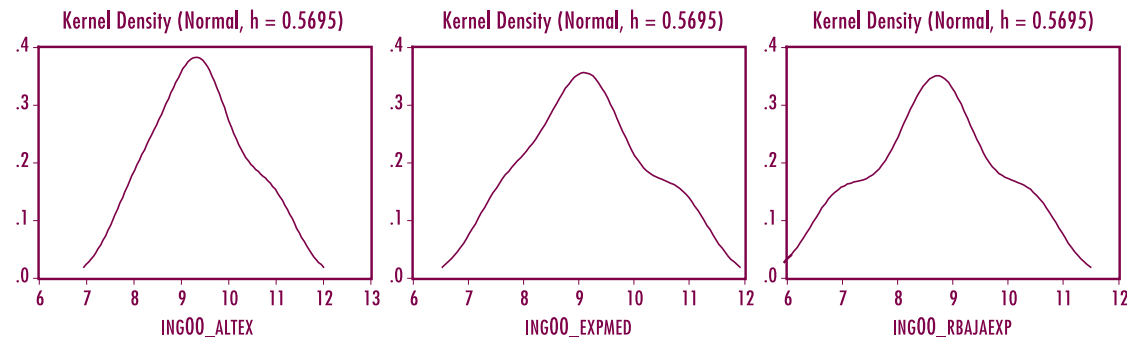

Fuente: Elaboración propia. 
menor incremento en el ingreso es AEG ( $\$ 851$ entre 1990 y 1995). El incremento en la región BEG fue de $\$ 891$. En cuanto al aspecto de la forma de la distribución, tenemos que en 1995 la concentración es mayor en el caso de la región EMG.

En el año 2000, los valores de las modas de cada una de las distribuciones son los siguientes: la región AEG tiene su moda en $\$ 10,938$, la región EMG en $\$ 8,104$ y la moda de la región BEG es de $\$ 6,003$. Hay incrementos en los niveles de ingreso promedio con respecto al año 1995 en todas las regiones. La región con un mayor incremento en el ingreso promedio es ahora la región AEG ( $\$ 1,983$ entre 1995 y 2000) seguida por la región BEG $(\$ 1,088)$ y finalmente la región EMG $(\$ 772)$. No obstante, la concentración en función de la forma distribucional ha seguido incrementándose en las tres regiones.

Gráfica 9

Funciones de distribución regional del ingreso, 2004
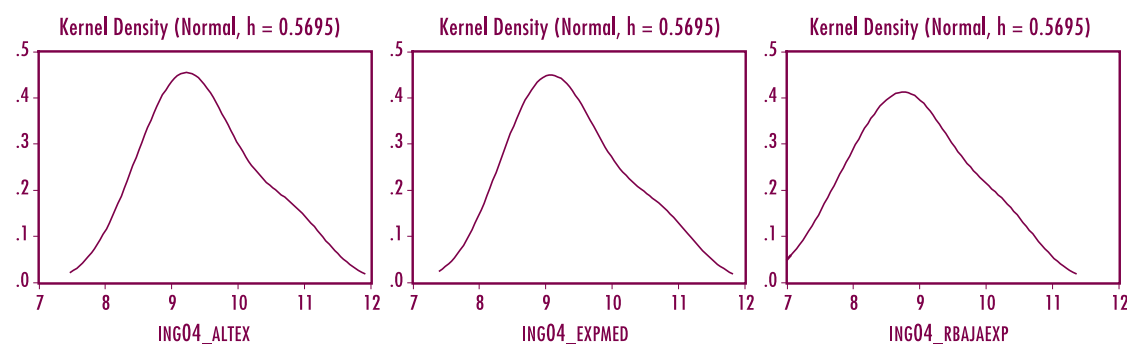

Fuente: Elaboración propia.

La gráfica 9 muestra las funciones regionales de distribución del ingreso en el año 2004. La región AEG tiene una moda en $\$ 12,058$ que indica el promedio de ingresos. La región EMG tiene su moda en $\$ 8,955$ y la región BEG en $\$ 6,634$. El ingreso de las tres regiones aumentó entre los años 2000 y 2004. Gráficamente las funciones se han desplazado a la derecha sobre el eje que representa el nivel de ingreso. La región que presentó un incremento mayor en su ingreso promedio fue la AEG ( $\$ 1,150$ entre 2000 y 2004) seguida por la región EMG $(\$ 851)$ y por la región BEG (\$631). La concentración parece haber disminuido en las tres regiones, pues se ha ensanchado el área que se encuentra próxima al valor de la moda en los tres casos. 


\section{Cambios en la distribución}

del ingreso y niveles de desigualdad

La desigualdad en términos de la dispersión es uno de los aspectos más importantes a analizar de la distribución del ingreso. La gran cantidad de literatura empírica y teórica han producido un número sustancial de medidas. ${ }^{12}$ Aquí revisamos dos de los índices más aceptados y utilizados en la literatura, y en la próxima sección reportamos sus resultados.

\section{El índice de Gini}

El índice de Gini parte de la base de una distribución dada del ingreso, y de ahí se pueden conocer las proporciones del mismo que obtienen los diferentes estratos poblacionales. Se calcula a partir de la curva de Lorenz que representa una distribución teórica cuando el ingreso se asigna de forma equitativa entre los miembros de la población. La fórmula utilizada en nuestro caso es la siguiente (Cortés y Ruvalcaba, 1984):

donde:

$$
G=\frac{\sum_{i=1}^{n-1}\left(P_{i}-Q_{i}\right)}{\sum_{i=1}^{n-1} P_{i}}
$$

$G=$ índice de Gini

$P_{i}=$ porcentaje acumulado de la población

$Q_{i}=$ porcentaje acumulado de ingreso

A partir de nuestras definiciones de ingreso, se tiene:

$$
G=\frac{\sum_{i=1}^{n-1}\left[(1 / 5)-\left(S_{i k t}\right)\right]}{\sum_{i=1}^{n-1} 1 / 5}
$$

El índice de Gini también puede expresarse en porcentaje cuando se multiplica por 100 .

12 Véase Cortés y Ruvalcaba (1984) y Cowell (1998) para una lista de medidas de desigualdad y sus potenciales desventajas. 


\section{El índice de Theil}

Por su parte, el índice de Theil surge de la teoría de la información. También se le conoce como índice de entropía. ${ }^{13} \mathrm{~A}$ partir de las definiciones de ingreso descritas anteriormente, el índice de Theil es el siguiente (Sala-i-Martin, 2002a):

$$
\begin{aligned}
& T_{t}=\frac{1}{N_{t}} \sum_{i=1}^{m} \sum_{j=1}^{N i t} \frac{y_{i j t}}{\overline{y_{t}}} \ln \left(\frac{y_{i j t}}{\overline{y_{t}}}\right) \\
& =\sum_{i=1}^{m} \sum_{k=1}^{5} \frac{N_{i t}}{N_{t}} \frac{S_{i k t}}{\overline{y_{t}}}\left[\ln \left(5 * S_{i k t} * y_{i t}\right)-\ln \left(\overline{y_{t}}\right)\right]
\end{aligned}
$$

donde:

$y_{i j t}=5 * s_{i k t} * y_{i t}$ es el ingreso por quintil poblacional,

$\overline{y_{t}}=\sum_{i=1}^{m} \frac{N_{i t}}{N_{t}} y_{i t}$ es el nivel de ingreso promedio,

$N_{t}=$ es la población total del país.

Al igual que en el caso del índice de Gini, este indicador cumple con la condición de Pigou-Dalton. ${ }^{14}$

Adicionalmente, en el caso del índice de Theil existe otra propiedad importante, la de descomposición. Desde el ámbito territorial (Conceicao y Ferreira, 2000; Rey, 2003), esto quiere decir que el índice de Theil puede descomponerse en partes que dan cuenta de la contribución individual que se tiene de la desigualdad entre las distintas regiones y la contribución a la desigualdad dentro de las mismas regiones. Ahora, si sumamos y restamos el término $\sum_{i=1}^{m} \frac{N_{i t} y_{i t}}{N_{t} \bar{y}_{t}} \ln \left(y_{i t}\right)$ a la ecuación (8), se
tiene:

$$
T=\sum_{i=1}^{m} \frac{N_{i t} y_{i j t}}{N_{t} \overline{y_{t}}} \ln \left(\frac{y_{i t}}{\overline{y_{t}}}\right)+\sum_{i=i}^{m} \frac{N_{i t} y_{i t}}{N_{t} \overline{y_{t}}} T_{i t}
$$

13 En general, la entropía es una medida que permite evaluar la degradación de un sistema, en términos de alguna variable.

14 Esta condición establece que un buen índice que captura la medición de la desigualdad debe incrementarse en respuesta a una transferencia de una persona pobre a una persona rica, aun y cuando el ingreso promedio de toda la población se mantenga en el mismo nivel. En otras palabras, el índice debe incrementarse cuando la desigualdad se aumenta, y viceversa. Véase Cowell (1998). 
donde $T_{i t}=\frac{1}{N_{i t}} \sum_{j=1}^{N_{i t}} \frac{y_{i j t}}{y_{i t}}\left[\ln \left(y_{i j t}\right)-\ln \left(y_{i t}\right)\right]$ es el índice de desigualdad de Theil para la región $i$ en el tiempo $t$. A partir de las definiciones de proporción del ingreso, el índice de Theil regional es:

$$
T_{i t}=\sum_{i=1}^{5} s_{i k t}\left[\ln \left(5 * s_{i k t} * y_{i t}\right)-\ln \left(y_{i t}\right)\right]
$$

Por tanto, la descomposición del índice de Theil puede resumirse de la siguiente forma:

$$
T_{t}=T_{E}+T_{D}
$$

donde:

$T_{E}=$ índice de Theil inter-regional,

$T_{D}=$ índice de Theil intra-regional

\section{Funciones de densidad y desigualdad regional: resultados}

En este apartado examinamos la evolución de la desigualdad en las regiones de México. ${ }^{15}$ En el siguiente cuadro se presentan las estimaciones de ambos coeficientes para la economía mexicana en su conjunto. ${ }^{16}$

Cuadro 3

Evolución de la desigualdad en México, 1990-2004

\begin{tabular}{ccccc}
\hline Indice & 1990 & 1995 & 2000 & 2004 \\
\hline Gini & 0.5316 & 0.5561 & 0.6162 & 0.5171 \\
Theil & 0.3383 & 0.3664 & 0.4634 & 0.3284 \\
\hline
\end{tabular}

Fuente: Cálculos propios

A partir del cuadro 3 se observa una tendencia al incremento de la desigualdad en el país medida por los dos índices. Entre 1990 y 2000, la desigualdad aumentó considerablemente. El índice de Gini pasó de 0.5316 a 0.6162 y el índice de Theil

15 Es necesario recordar que el índice de Gini toma valores entre 0 y 1 , este último indicando máxima desigualdad. Por su parte, el índice de Theil toma valores entre 0 y $\ln (n)$, también indicando este último la máxima desigualdad.

16 Los valores del índice de Gini fueron calculados a partir de la ecuación (7) y los del índice de Theil se estimaron con la ecuación (10). 
lo hizo de 0.3383 a 0.4634 . Esto indica un empeoramiento en la asignación del ingreso en el país.

Sin embargo, es notable la reducción en la desigualdad entre los años 2000 y 2004. La década de los noventa fue difícil desde la perspectiva de la desigualdad. La crisis de 1995 contribuyó, sin duda, al deterioro de las condiciones de vida de los estratos inferiores de la población. Aun con el crecimiento experimentado en los últimos años de dicha década, no se redujo la desigualdad. A continuación, revisará el impacto de la desigualdad en las distintas regiones definidas para este estudio. En este caso se han utilizado las ecuaciones (10) y (11).

Cuadro 4

Índice de Gini. Regiones de México, 1990-2004

\begin{tabular}{lllll}
\hline \multicolumn{1}{c}{ Región } & 1990 & 1995 & 2000 & 2004 \\
\hline Alta Exposición & 0.5185 & 0.5493 & 0.5839 & 0.5114 \\
Exposición Media & 0.5383 & 0.5571 & 0.6309 & 0.5199 \\
Baja Exposición & 0.5423 & 0.5702 & 0.6415 & 0.5251 \\
\hline
\end{tabular}

Fuente: Cálculos propios.

La tendencia al aumento en el valor del índice de Gini se mantiene en las regiones del país en el periodo comprendido entre 1990 y 2000. Sin embargo, cuando se revisan los niveles de desigualdad, se encuentra que en todos los años la desigualdad es más alta en la región BEG. La región AEG tiene los niveles de desigualdad más bajos de las tres regiones. Asimismo, en 2004 se reduce la desigualdad regional en México.

La desigualdad sigue siendo una característica importante y negativa de la economía mexicana, incluso cuando se destaca sobre el aspecto espacial de la misma.

Cuadro 5

Índice de Theil. Regiones de México 1990-2004

\begin{tabular}{lllll}
\hline Región & 1990 & 1995 & 2000 & 2004 \\
\hline Alta Exposición & 0.3067 & 0.3458 & 0.3895 & 0.2994 \\
Exposición Media & 0.3332 & 0.3655 & 0.4586 & 0.3084 \\
Baja Exposición & 0.3307 & 0.3641 & 0.4766 & 0.3074 \\
\hline
\end{tabular}

Fuente: Cálculos propios.

Los resultados que se muestran en el cuadro 5 confirman lo antes mencionado: la región AEG presenta los niveles de desigualdad más bajos de las tres regiones 
estudiadas. Aparentemente, los niveles de desigualdad son similares en las regiones BEG y EMG en 1990 y 1995. Ya en 2000 la región BEG presenta el valor más elevado del índice Theil. Otra vez, en 2004 el valor del índice disminuye en las tres regiones, denotando una reducción en la desigualdad.

Es importante conocer la contribución de las diferentes unidades de análisis sobre la desigualdad total. Para esto, se trata de responder a las siguientes preguntas: ¿qué tanto de la desigualdad se explica por la desigualdad que existe ENTRE las regiones del país?, y, por otra parte, ¿qué tanto de la desigualdad es explicada por la desigualdad que hay DENTRO de las regiones del país?

Por tanto, es necesario desagregar el índice de Theil ${ }^{17}$ en sus dos componentes principales: el componente intra-regional y el componente inter-regional ${ }^{18}$. Los resultados se muestran a continuación:

Cuadro 6

Descomposición del Índice de Theil, México 1990-2004

\begin{tabular}{ccccc}
\hline Componente & 1990 & 1995 & 2000 & 2004 \\
\hline Intra Regional & 0.3234 & 0.3532 & 0.4426 & 0.3045 \\
Inter Regional & 0.0149 & 0.0132 & 0.0208 & 0.0239 \\
\hline
\end{tabular}

Fuente: Cálculos propios.

El componente intra-regional es en todos los casos el que mayormente explica la desigualdad de las regiones de México. La tendencia al incremento en el componente intra-regional indica que la puesta en marcha del TLCAN no contribuyó en la década de los noventa a una reducción en la misma. El componente inter-regional muestra un patrón de divergencia económica entre 1990 y 1995. Este patrón cambió entre 1995 y 2000 y se ha prolongado hasta 2004. Sin embargo, es claro que el nivel al que se da esta convergencia de ingresos es insuficiente para compensar el rezago con el que cuenta la región BEG con respecto de EMG y sobre todo de AEG.

17 No todos los índices de desigualdad se pueden descomponer en partes. Cowell (1998) muestra que entre los índices que se pueden descomponer sin tener problemas de significación estadística están los índices de entropía, a los cuales pertenece el índice de Theil. Por esta razón es que en nuestro ejercicio se descompuso dicho índice.

18 El componente inter-regional muestra el grado al cual los niveles de ingreso tienden a converger unos con otros. El componente intra-regional destaca las disparidades dentro de cada una de las regiones. 


\section{Conclusiones}

Un alto grado de desigualdad en el ingreso ha sido una característica persistente y problemática de la economía mexicana en los años recientes. Sin embargo, los resultados muestran que el nivel de desigualdad no es similar en las distintas regiones del país. En general, se encuentra que los niveles de desigualdad son menores en el caso de las entidades que han estado mayormente expuestas a la globalización económica.

En primera instancia, el análisis de la convergencia económica permite afirmar que tal y como se ha documentado en otros estudios, dicho proceso se revirtió a finales de la década de los ochenta, para no volverse a recuperar. Incluso la tasa de divergencia económica regional se incrementó en el periodo de la apertura comercial. Ello permite afirmar que también la divergencia es una característica de las economías regionales en México. En estos términos, sólo las entidades dotadas de una mejor infraestructura y mejor ubicadas geográficamente han tomado ventaja de las nuevas fuentes de crecimiento ofrecidas por la apertura comercial. Estas entidades tienden a estar más vinculadas económicamente con el sur de Estados Unidos que con el sur de México. Los costos de los efectos ocasionados por los diversos programas de ajuste se han traducido en una ralentización del crecimiento de las economías estatales. Lo anterior parece reforzar la idea de que la unidad de análisis cuando se estudia la hipótesis de la convergencia y el comercio internacional debe ser la región, en lugar del país.

Adicionalmente, aun y cuando las funciones de distribución del ingreso presentan movimientos positivos en el sentido de que el ingreso se ha incrementado, ello no se ha traducido en mejoras inequívocas de las condiciones de vida de la población. Las disparidades dentro de las regiones explican la mayor parte del patrón regional de la desigualdad.

Desde una perspectiva teórica, se está ante la verificación de un proceso circular y acumulativo: hay una región que se ha adaptado mejor a los cambios provenientes del exterior en cuanto al arreglo tecnológico y de acceso al mercado. Los efectos "retardadores" con los que cuenta esta región son la infraestructura necesaria para un mejor acceso al mercado externo y las ventajas de localización con respecto a dicho mercado. En este sentido, estos mecanismos han ido generando beneficios que no se transmiten hacia el resto de las regiones, ocasionando al mismo tiempo que la brecha que separa a las economías regionales se haya ido ensanchando.

Finalmente, el grado de exposición regional a la globalización establece un efecto diferenciado y polarizante de la economía nacional: hay una región que se ha visto más beneficiada de la inserción de la economía a la competencia internacional en detrimento de las otras regiones. La apertura ha conducido a la economía mexicana hacia la polarización. 


\section{Bibliografía}

Aspe, Pedro, El camino mexicano de la transformación económica, México, FCE, Textos de Economía, $2^{a}$ ed., 1993.

Barro, Robert y Xavier Sala-i-Martin, "Convergence", en Journal of Political Economy, vol. 100, núm. 2, pp. 223-251, Chicago, 1992.

Burguer, John D., "U or Inverted U? That is The Question", en Review of Development Economics, vol. 5, núm. 1, pp. 151-156. Aimes, 2001.

Cárdenas-Rodríguez, Óscar, Gabriel González-Konig, Diana Ojeda-Revah y Quentin Wodon, "Do Indigenous People Benefit From Poverty Programs? Evidence From Mexico's 2000 Census", en Estudios Económicos 37, vol. 19, núm. 1, pp. 125-135, México, 2004.

Chiquiar, Daniel, "Why Mexico's Regional Convergence Broke Down", en Journal of Development Economics, vol.77 pp. 257-275. Cambridge, 2005.

Consejo Nacional de Población, Indicadores Demográficos Básicos Datos de Población 1970-2005, México, CONAPO, 2005.

Conceicao, Pedro y Pedro Ferreira, "A Young's Person Guide to the Theil Index: Suggesting Intuitive Interpretations and Exploring Analytical Applications", en The University of Texas Inequality Project Working Paper Series, núm. 14, Austin, 2000.

Cortés, Fernando y Rosa María Ruvalcaba, Técnicas estadísticas para el estudio de la desigualdad social, $1^{\mathrm{a}}$ ed., México, El Colegio de México y la Facultad Latinoamericana de Ciencias Sociales, 1984

Cowell, Frank, "The Measurement of Inequality", Discussion Paper No. DARP/36, STICERD, London School of Economics and Political Science, Londres, 1998.

Cuadros, Ana, Vicente Orts y Maite Alguacil, "Openness and Growth: Re-Examining Foreign Direct Investment, Trade and Output Linkages in Latin America", en The Journal of Development Studies, vol. 40, núm. 4, pp. 167-192, Cambridge, 2004.

Esquivel, Gerardo, "Convergencia Regional en México 1940-1995", en El Trimestre Económico, vol. 66, núm. 4, pp. 725-761, México, FCE, 1999.

y Miguel Messmacher, "Sources of Regional (non) Convergence in Mexico", mimeo, México, El Colegio de México, 2002.
Fuentes, Noé Arón, "Apertura Comercial y Divergencia Económica Regional", Departamento de Estudios Económicos, Tijuana, El Colegio de la Frontera Norte, 2001.

Hanson, Gordon, "Globalization, Labor Income and Poverty in Mexico", Working Paper No. 11027, National Bureau of Economic Research Working Paper Series, Washington, 2005a.

"Emigration, Labor Supply and Earnings in Mexico", Working Paper No. 11412, National Bureau of Economic Research Working Paper Series, Washington, 2005b.

Instituto Nacional de Estadística, Geografía e Informática, Sistema de Cuentas Nacionales de México (SCNM). Producto Interno Bruto por Entidad Federativa 1980-2004, México, INEGI, 2005.

, XI Censo General de Población y Vivienda, 1990, México, INEGI, 1991.

, XII Censo General de Población y Vivienda,2000, México, INEGI, 2001.

Jenkins, Stephen P. y Philippe Van Kerm, "Accounting for Income Distribution Trends: A Density Function Decomposition Approach", IRISS Working Paper 2004-7, Luxemburg, CEPS/INSTEAD, Differdange, G.-D., 2004.

Levin, Richard y David Rubin, Estadística para administración y economía, $7^{\mathrm{a}}$ ed., México, Pearson, 2004.

Messmacher, Miguel, "Desigualdad Regional en México. El TLCAN y otras Reformas Estructurales", Documento de Investigación no. 2000-4, México, Banco de México, 2000.

Quah, Danny T., "Twin Peaks: Growth and Convergence in Models of Distribution Analysis", en The Economic Journal, núm. 106 (julio), pp. 1045-1055, Oxford, 1996. "Empirics for Growth and Distribution: Specification, Polarization and Convergence Clubs", Discussion Paper No. 324, Center for Economic Performance, London School of Economics and Political Science, pp. 1-70, Londres, 1997.

Ramírez, Miguel D., "Foreign Direct Investment in Mexico During the 1990's: An Empirical Assessment", en Eastern Economic Journal, vol. 28, núm. 3, pp. 409-423, Nueva Rochelle, 2002. 
Rey, Serge, "Spatial Analysis of Regional Income Inequality", en Michael F. Goodchild y Donald G. Danielle (editores), Best Practices in Spatially Integrated Social Sciences, Cambridge, Oxford University Press, 2003.

Ruiz-Durán, Clemente, Dimensión territorial del desarrollo económico en México, México, Facultad de Economía, UNAM, 2004.

Sala-i-Martin, Xavier, "The Classical Approach to Convergence Analysis", en The Economic Journal, núm. 106 (julio), pp. 1019-1036, Oxford, 1996.

"The Disturbing "Rise" of Global Income Inequality", Working Paper No. 8904, National Bureau of Economic Re- search Working Paper Series, Washington, 2002a.

,"The World Distribution of Income (Estimated From Individual Country Distributions)", Working Paper No. 8993, National Bureau of Economic Research Working Paper Series, Washington, 2002b.

Silverman, Bernard W., Density Estimation for Statistics and Data Analysis, Boca Raton, Chapman and Hall, 1986.

Unger, Kurt, "Regional Economic Development and Mexican Out-Migration", Working Paper No. 11432, National Bureau of Economic Research Working Paper Series, Washington, 2005. 